\title{
The Heart is a Target Organ in Offspring Rats Due to Maternal Hypertension
}

\author{
Sonia Regina J urado ${ }^{1 *}$, Roberto J orge da Silva Franco ${ }^{2}$, Antonia Dalla Pria \\ Bankoff ${ }^{3}$, Andrea Sanchez ${ }^{3}$ \\ ${ }^{1}$ Federal University of Mato Grosso do Sul, Brazil \\ ${ }^{2}$ Universidade Estadual Paulista Julio de Mesquita Filho, Brazil \\ ${ }^{3}$ Federal University of Mato Grosso do Sul, Brazil
}

Received: October 29, 2013; Accepted: December 10, 2013; Published: December 16, 2013

*Corresponding author: Sonia Regina Jurado, Federal University of Mato Grosso do Sul, Tres Lagoas, Mato Grosso do Sul, Avenue Ranulpho Marques Leal, Brazil, Tel: +55 67-3509-3714,E-mail: srjurado@bol.com.br

\begin{abstract}
Purpose: The aim of this study was to analyze the effect of hereditary hypertension or induced by chronic nitric oxide inhibition during pregnancy on the structural changes in rats fetal and neonate coronary microvessels, aorta and myocardial collagen content.

Methods: Total nine sub-groups allocated from three main groups of fetuses $\left(20^{\text {th }} d\right)$ and newborns ( $2^{\text {nd }}$ and $\left.15^{\text {th }} d\right)$ offspring's from normotensive mothers (C, control), SHR (spontaneously hypertensive rats) and L-NAME ( $\mathrm{N}^{\omega}$-Nitro-L-Arginine Methyl Ester) were studied. Heart and aorta sections were stained with hematoxylin and eosin, Mallory's thichrome, Picrosirius red and periodic acid-Schiff reagent. Pro-Plus image analysis system was used to assess the thickness of aorta and myocardial vessels. The tunica media inner and outer border was traced in each microvessel (external diameter $<50 \mu \mathrm{m}$ ) image at x100 and, in aorta at x400 magnification, and areas encircled by tracings were calculated.
\end{abstract}

Results: Aorta wall area and wall-to-lumen ratio increased in hypertensive animals in all ages. The number of elastic lamellae was increased in hypertension (L-NAME: $7.10 \pm 0.18, p<0.05$ and SHR: $7.45 \pm 0.17, \mathrm{p}<0.05)$ at $2^{\text {nd }} d$ compared to $C(7.60 \pm 0.17)$. The microvessels wall area was greater in hypertensive offspring at $15^{\text {th }}$ d. Lumen area and external perimeter decreased in both hypertension models at $15^{\text {th }} \mathrm{d}$. Wall-to-lumen ratio increased in hypertensive at $2^{\text {nd }}$ and $15^{\text {th }} d$. Collagen content (\%) was higher in SHR at $2^{\text {nd }} d$ $(0.74 \pm 0.02, \mathrm{p}<0.05)$ and $15^{\text {th }} \mathrm{d}(2.53 \pm 0.04, \mathrm{p}<0.05)$ and in L-NAME at $15^{\text {th }}(2.25 \pm 0.04, p<0.05)$ versus $C\left(0.61 \pm 0.02\right.$ at $2^{\text {nd }} d$ and $2.12 \pm 0.05$ at $\left.15^{\text {th }} \mathrm{d}\right)$.

Conclusion: Maternal hypertension, as in the model L-NAME and SHR, caused microvasculature remodeling in heart the neonate at 2 and 15 days.

Keywords: Nitric oxide; Microvasculature; Aorta; Myocardium; Hypertension; Pregnancy; Morphometry; Fetus; Newborn; Rat

Abbreviations: ACE: Angiotension I Converting Enzyme; C20: Control group at 20 post conception days; C2: Control group at 2 post-natal days; C15: Control group at 15 post-natal days; g: grams; Kg: Kilograms; L20: L-NAME group at 20 post conception days; L2: L-NAME group at 2 post-natal days; L15: L-NAME group at 15 post-natal days; L-NAME: $\mathrm{N}^{\omega}$-Nitro-L-Arginine Methyl Ester; mg: milligrams; NO: Nitric Oxide; NOS: Nitric Oxide Synthase; S20: SHR group at 20 post conception days; S2: SHR group at 2 post-natal days; S15: SHR group at 15 post-natal days; SHR: Spontaneously Hypertensive Rats; $\mu \mathrm{m}$ : micrometers

\section{Introduction}

Nitric oxide (NO) is synthesized from L-arginine by the constitutive NO synthase in vascular endothelial cells, and plays an important role in the regulation of blood pressure and coronary vasomotion [1]. Normal pregnancy is associated with major adaptations in maternal cardiovascular function, which help the woman to accommodate the growing fetus. Although there is an increase of maternal blood pressure volume and cardiac output as well, the systemic blood pressure actually declines during pregnancy [2]. Furthermore, pressure responsiveness or vascular reactivity to several vasoconstrictors is attenuated. This is probably due to the potential contribution of nitric oxide to the vasodilator phenomena of pregnancy [3]. Preeclampsia constitutes the syndrome of vasoconstriction with elevated arterial blood pressure, edema, proteinuria, kidney and liver dysfunction, and intrauterine growth retardation [4,5]. The vascular endothelium is stimulated during pregnancy to release increased amounts of NO and the abnormality in the L-arginineNO pathway may play a role in the etiology of preeclampsia [3].

It is known that the administration of L-NAME $\left(\mathrm{N}^{\omega}\right.$-NitroL-Arginine Methyl Ester) in adult rats promotes arterial hypertension [6,7], cardiac hypertrophy, myocytic necrosis, perivascular and interstitial fibrosis and microvascular lumen occlusion [8,9] and cardiomyocyte apoptosis [10]. However, low dose of L-NAME administrated in rats under prolonged period caused arterial hypertension accompanied by a significant reduction in cardiac weight and cardiomyocyte size [11]. In pregnant rats, this nitric oxide synthesis inhibitor causes fetal growth restriction by a reduction in cellular proliferation due 
to induction of apoptosis [12], reducing the body weight and causing hemorrhagic necrosis of neonate's hind limbs $[13,14]$.

The role of nitric oxide on fetal cardiovascular development is only partially known. Mice with endothelial nitric oxide synthase deficiency had bicuspid aortic valves [15], increased cardiomyocyte apoptosis, congenital septal defects [16], fetal growth restriction and reduced survival [17].

The NO-generation is required for cardiomyogenesis since NO synthase inhibitors prevent the maturation of terminally differentiated cardiomyocytes in vitro and the number of differentiated cardiomyocytes is significantly reduced [18].

The purpose of this work was to determine whether the fetus and neonate myocardium microvasculature and aorta as well as myocardial collagen content were affected due to oxide nitric synthase inhibition during pregnancy in rats.

\section{Material and Methods}

Thirty females 14-16-week-old Wistar and fifteen spontaneously hypertensive rats (SHR) with a body weight of 200-250 g were mated with male rats. Day 1 of pregnancy was determined by the presence of spermatozoa in vaginal smear. The animals were housed under controlled lighting $112: 12 \mathrm{~h}$ light-dark cycle) and temperature $\left(21 \pm 3^{\circ} \mathrm{C}\right)$. The Wistar dams were randomly assigned to two groups of 15 rats each: control and L-NAME ( $\mathrm{N}^{\omega}$-Nitro-L-Arginine Methyl Ester). The L-NAME animals received the NO synthase inhibitor (hydrochloride, L-NAME, Sigma, St Louis, MO, lot 70H7703) in drinking water (12 mg/day/rat), throughout the pregnancy (21days). The rat arterial pressure was evaluated by tail cuff plethysmography at the beginning (on day 1 of gestation) and end of gestation (on day 21 of gestation). The average of three successive measurements was taken as the mean systolic pressure value. A total of nine subgroups of fetuses and pups were studied from three main groups (Control, L-NAME and SHR). Fifteen fetuses were separated into three sub-groups of five each: C20, L20 and S20 (C: control, L: L-NAME, S: SHR; 20: at 20 post conception days). Thirty newborns also were allocated in the following sub-groups of five each: C2, C15, L2, L15, S2, S15 (2 and 15: at 2 and 15 post-natal days). They were sacrificed under pentobarbital anesthesia. All handling and procedures observed minimum animal suffering, and procedures and experimental protocols were approved by the Ethical Committee of the Botucatu Medical School - UNESP.

The heart and aorta were dissected, fixed for 24 hours by immersion in buffered formalin $10 \%$ (pH 7.2). The samples were dehydrated in ethanol, embedded in paraplast and sectioned 5-6 $\mu \mathrm{m}$ thick. Sections were stained with hematoxylin and eosin, Masson's trichrome, picrosirius red and periodic acid-Schiff (PAS) reagent.

\section{Quantitative assessment of myocardial collagen}

It was analyzed thirty fields of view from each heart section ( $6 \mu \mathrm{m}$ thick) stained with picrosirius red to provide a range of collagen contents (types I and III). Specifically, we examined tissue from the free wall of the left ventricle, right ventricle, and intraventricular septum from newborn at 2 and 15 days. The areas of the myocardium that contain collagen, such the perivascular space, were excluded. It was not performed the collagen content in fetal heart because the collagen network was still in development. The myocardial collagen content was assessed by digital image analysis using Pro-Plus analysis system (Media Cybernetics, USA) and polarized light microscopy at level $\mathrm{x} 40$. At polarized light, picrosirius red stained sections are viewed in brightfield, type I collagen appears red and type III green. This technique provides a convenient and a quantitative histologic approach that can also be used to quantify collagen content [19].

\section{Cardiac microvasculature and aorta morphometry}

Heart and aorta sections stained by hematoxylin-eosin were observed through a light microscope (Leica DMLB) and their images were recorded by a video camera and then loaded into a Pro-Plus analysis system (Media Cybernetics, USA). All vessels in the section had their tunica media, lumen and outer border traced at x400 magnification. Aorta section was studied at x100 magnification. The following variables for each blood vessel were then calculated by the Pro-Plus analysis system: total vessel area, vessel wall area, vessel lumen area, wall-to-lumen ratio (the area of the vessel wall divided by the area of the blood vessel lumen), minimum and maximal diameters and external perimeter. More than 150 blood vessels were analyzed in each group. To compare the myocardial microvasculature tunica media - capillaries and arterioles, minimum diameter ranging from 5 to $50 \mu \mathrm{m}$, only transverse sectional images (minimum diameter/maximum diameter $>0.60$ ) were considered.

The aortas of newborns at 2 and 15 days also had the number of elastic lamellae in each of four quadrants counted using light microscopy.

\section{Statistics}

Data are expressed as mean \pm SD. Comparisons of the maternal arterial pressure, fetal and neonatal length, body and cardiac weights and relative cardiac weight were performed by one-way ANOVA. Comparisons of the vessel external perimeter, external area, lumen area, wall area, wall-to-lumen ratio, collagen content and number of elastic lamellae were performed by two-way (treatment $x$ age) ANOVA followed by multiple comparisons tests. A probability of p 0.05 was considered statistically significant.

\section{Results}

\section{Maternal arterial pressure}

L-NAME and SHR pregnant rats showed higher arterial pressure relative to dams normotensive (Figure 1). An insignificant fall in blood pressure was observed in the Wistar $(98.8 \pm 4.6 \mathrm{mmHg})$ and SHR $(178 \pm 5.2 \mathrm{mmHg})$ dams at the end of gestation when compared at the beginning $(102.0 \pm 5.2 \mathrm{mmHg}$ and $179.0 \pm 6.4 \mathrm{mmHg}$, respectively). 


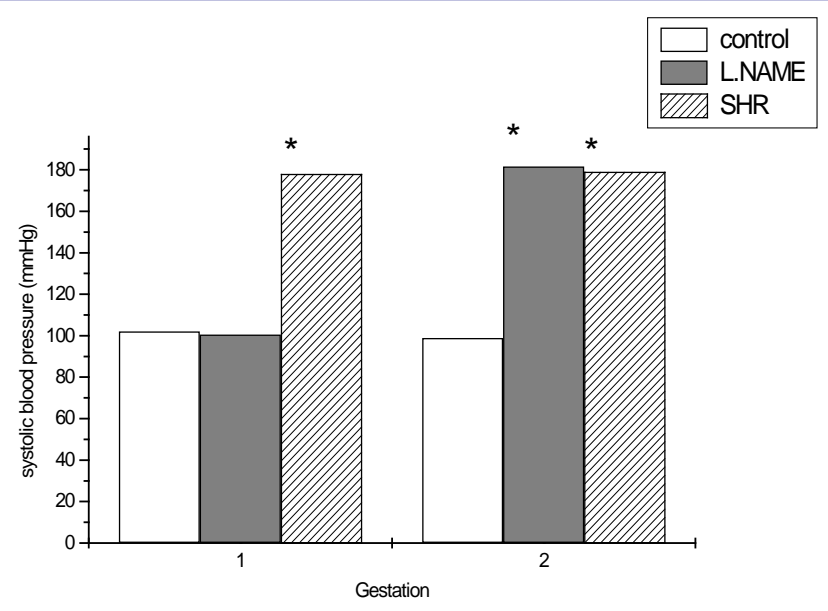

Notes: Blood pressure at beginning (1) and end (2) of gestation in the control, L-NAME and SHR dams. "P $<0.05$ vs control group by one-way followed by the multiple comparison test

Figure 1: Bar graph of systolic blood pressure of the pregnant rats.

The L-NAME administration during pregnancy promoted hind-limbs disruptions in pups of three litters. These consisted of small damages in the phalanxes up to hemorrhagic necrosis of one or both legs. We did not undertake histologic analysis of these regions because it was not the aim of the study.

\section{Vertice-coccyx length, body weight and cardiac weight}

As described in Table 1, fetal (20 days of gestation) and newborn body weights of L-NAME and SHR were lower than that of controls. Also, L-NAME and SHR pups were smaller than their counterparts, except for L-NAME at 15 days of age. There was no significant difference of cardiac weights for fetal groups. However, a statistically significant difference of cardiac weights was noted among Control, L-NAME and SHR groups at 2 and 15 post-natal days.

When expressed as \% body weight, the relative cardiac weight was significantly greater in SHR fetuses $(\mathrm{P}<0.01)$, L-NAME and SHR newborns at 15 post-natal days $(\mathrm{P}<0.001$ and $\mathrm{P}<0.01$, respectively) than control animals. However, this parameter decreased significantly in the newborn L-NAME at 2 days relative to the control group at the same age $(\mathrm{P}<0.001)$ (Table 1$)$.

\section{Cardiac and aorta histology}

Compared with the control group, SHR and L-NAME did not show areas of myocardial fibrosis (Figures $2 \mathrm{a}$ and $2 \mathrm{~d}, 2 \mathrm{~b}$ and $2 \mathrm{e}$, respectively). At the age of 2 days, was observed with frequency cardiomyocytes in division and apoptosis cells for all the groups (Figure $2 \mathrm{~b}, 2 \mathrm{~d}$ and $2 \mathrm{e}$ ). The myocardium of L-NAME newborns at 15 days of age presented more quantity of collagen around the vessels and cardiac interstitium (Figure $2 \mathrm{f}$ ) relative to controls at the same age (Figure 2c).

In rat fetus at 20 days, the major part of the aorta wall is tunica media, which consists of several layers of immature smooth
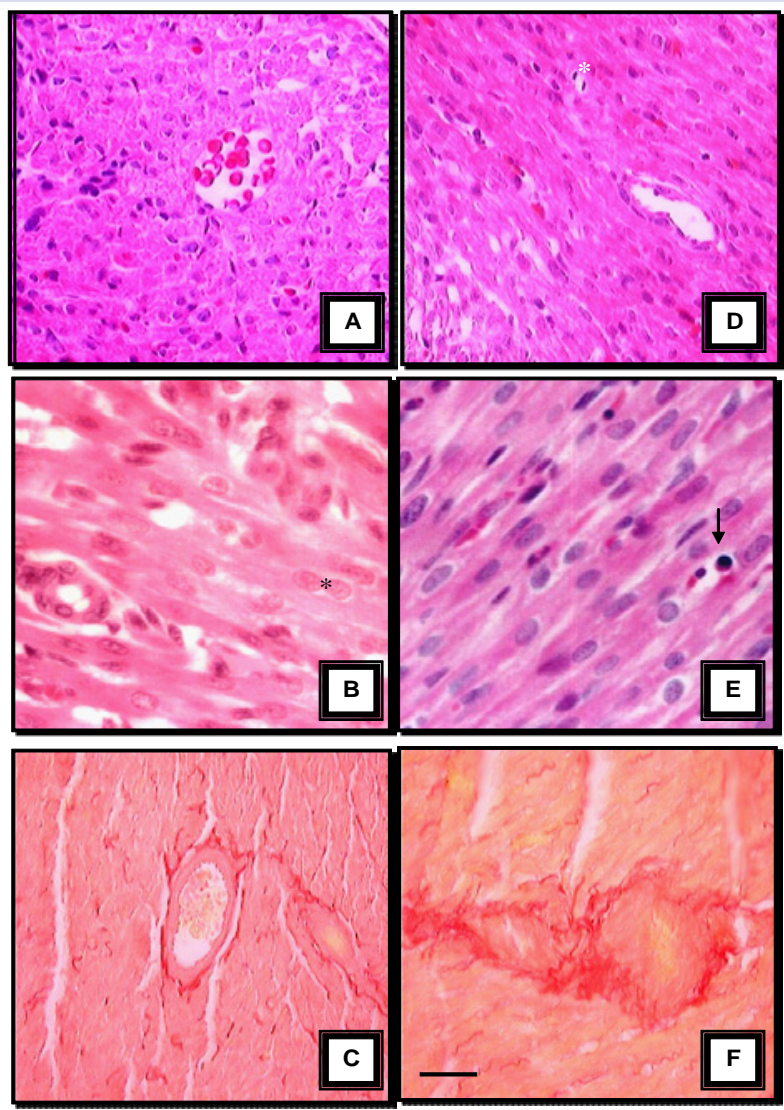

Notes: Myocardium of control (A, B and C), SHR (D) and L-NAME (E and F) newborns stained by hematoxylin-eosin and picrosirius red. Asterisk indicates cardiomyocytes in division. Arrow indicates apoptotic cells. Scale bar $=10 \mu \mathrm{m}$

Figure 2: Photomicrographs of the myocardium of control, SHR and L-NAME newborns.

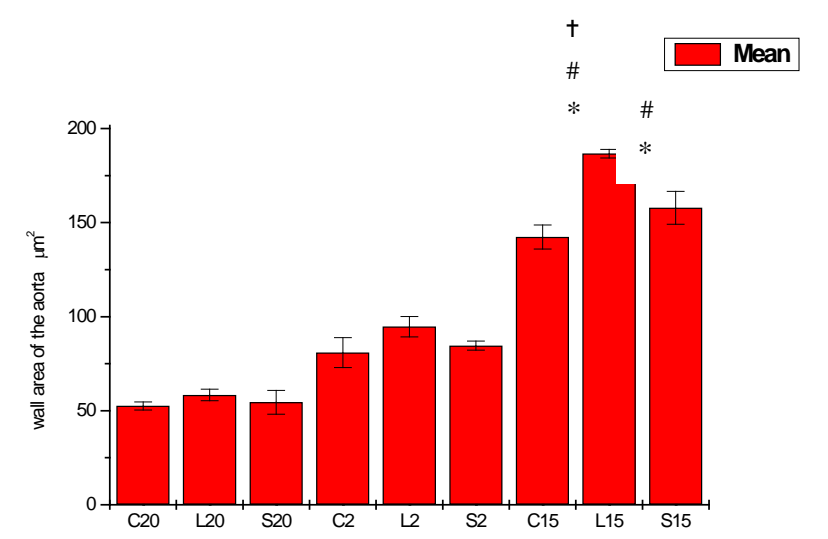

Notes: ${ }^{*} \mathrm{P}<0.05$ vs $\mathrm{C} 20,{ }^{\#} \mathrm{P}<0.05$ vs $\mathrm{C} 2,{ }^{+} \mathrm{P}<0.05$ vs $\mathrm{C} 15$ by two way ANOVA followed by multiple comparison tests. C20, L20 and S20: fetuses at 20 days of control (C), L-NAME (L) and SHR (S) groups. C2, L2 and S2: control, L-NAME and SHR newborns at 2 post-natal days. C15, L15 and S15: control, L-NAME and SHR newborns at 15 post-natal days. Values are means $\pm \mathrm{SD} ; \mathrm{n}=5$ animals per group

Figure 3: Bar graph of the wall area of the aortas from control, L-NAME and SHR animals. 


\begin{tabular}{|c|c|c|c|c|c|}
\hline & & $\begin{array}{l}\text { V-C } \\
(\mathrm{mm})\end{array}$ & $\begin{array}{l}\text { Total Body } \\
\text { (g) }\end{array}$ & $\begin{array}{l}\text { Cardiac } \\
\text { (g) }\end{array}$ & $\begin{array}{l}\text { Cardiac/Body } \\
(\%)\end{array}$ \\
\hline \multirow{3}{*}{ Fetus } & Control & $3.222 \pm 0.048$ & 2.8150 .248 & $0.017 \pm 0.002$ & $0.404 \pm 0.050$ \\
\hline & L-NAME & $2.972 \pm 0.036^{*}$ & $2.138 \pm 0.267^{*}$ & $0.015 \pm 0.003$ & 0.3700 .104 \\
\hline & SHR & $2.958 \pm 0.086^{*}$ & $2.092 \pm 0.105^{*}$ & $0.015 \pm 0.002$ & $0.6020 .075^{*}$ \\
\hline \multirow[t]{2}{*}{ 2-day } & L-NAME & $4.370 \pm 0.077^{*}$ & $6.463 \pm 0.312^{*}$ & $0.034 \pm 0.003^{*}$ & $0.569 \pm 0.035^{*}$ \\
\hline & SHR & $4.290 \pm 0.318^{*}$ & $6.550 \pm 0.265^{*}$ & $0.048 \pm 0.006^{*}$ & $0.731 \pm 0.112$ \\
\hline \multirow[b]{2}{*}{ 15-day } & Control & $6.898 \pm 0.136$ & 29.8321 .315 & $0.135 \pm 0.008$ & $0.570 \pm 0.029$ \\
\hline & L-NAME & $7.466 \pm 0.192^{*}$ & $27.906 \pm 2.424$ & $0.163 \pm 0.013^{*}$ & $0.728 \pm 0.067^{*}$ \\
\hline
\end{tabular}

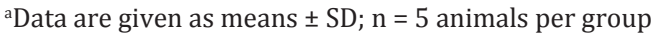

${ }^{*} \mathrm{P}<0.05$ vs controls by one-way ANOVA followed by the multiple comparison test

Table 1: Details of fetuses and newborns (control, L-NAME and SHR mothers) at 2 and 15 days ${ }^{\mathrm{a}}$.

muscle cells where the internal elastic membrane is observed. There were no differences among aortas of control, L-NAME and SHR fetuses. In newborn at 2 days, there was thickening of media for all groups, however the elastic lamellae were less individualized. In newborn at 15 days, the elastic lamellae were well developed.

\section{Aorta morphometry}

The wall area of the aorta was greater in hypertensive groups (L-NAME and SHR) than in control groups for all ages (Figure $3)$. The wall-to-lumen ratio increased significantly in L-NAME fetuses in relative to controls (Figure 4).

There was an increase in the number of elastic lamellae in L-NAME and SHR groups compared with control animals at 2 days, and stop increasing the number of elastic lamellae at 15 days comparing the same groups (Table 2).

\section{Cardiac microvasculature morphometry}

Representative examples of coronary vessels in the groups studied at 2 and 15 days are shown in Figure 5.

Total blood vessel area (external area) increased with postnatal cardiac growth, but there was a significant difference between control, L-NAME and SHR neonates only in the age of 15 days (Figure 6).

The wall area in the microvessels was greater in the L-NAME and SHR groups than in the control group, however significant difference was noted only at 15 post-natal days (Figure 7). Also, the lumen area and external perimeter decreased significantly in L-NAME and SHR newborn at 15 post-natal days when compared to controls at the same age.

The wall-to-lumen ratio increased significantly in newborns at 2 and 15 post-natal days of L-NAME and SHR groups relative to controls at the same ages (Figure 8).

\section{Collagen content}

The collagen content is summarized in Table 2. Collagen content was higher in the heart of SHR newborns at 2 days and L-NAME and SHR at 15 days.

\section{Discussion}

There are few data in literature about the importance of nitric oxide in the fetus and neonate development and growing. Our results agree with previous ones showing that inhibition of nitric oxide by L-NAME during the gestation disturbed the development of fetus limbs $[13,14]$.

Gardiner and collaborators [20] demonstrated that the initial hypertensive response after intravenous administration of L-NAME is accompanied by marked vasoconstriction in the hindquarter, mesenteric and renal vascular beds. Over time, both mesenteric and renal vasculature returns to baseline tone, whereas the hind limb circulation demonstrates a sustained vasoconstriction. This suggests that L-NAME crosses the placental barrier and affects the fetal nitric oxide synthesis, leading to cell death in the limbs because the NO has a role for in limb and digit development [21].

In this study, apoptotic cells were observed with a frequency in the myocardium of the all the groups at 2 post-natal days. Thus, apoptosis or programmed cell death takes place in situations of heart remodeling during or after the pathological processes $[22,23]$. Therefore, it is logical to believe that apoptosis may also occur in post-natal maturation of the heart and other tissues of the cardiovascular system, which have to adapt to their new hemodynamic role.

When expressed as \% body weight, the relative cardiac weight was significantly greater in SHR fetuses and L-NAME and SHR newborns at 15 post-natal days than control animals. The increase in relative cardiac weight of these animals may 


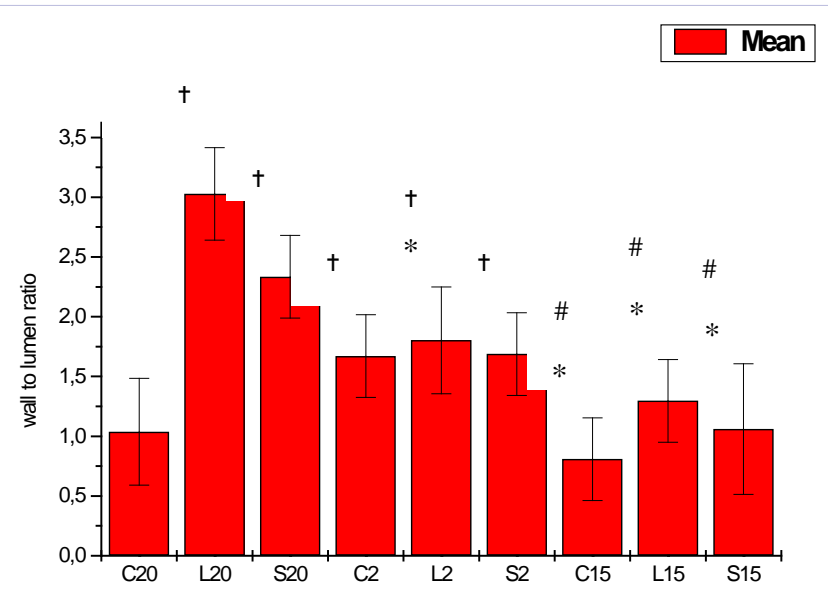

Notes: ${ }^{*} \mathrm{P}<0.05$ vs $\mathrm{C} 20,{ }^{*} \mathrm{P}<0.05$ vs $\mathrm{C} 2, \mathrm{P}<0.05$ vs $\mathrm{C} 15$ by two way ANOVA followed by multiple comparison tests. Abbreviations as in Figure 3

Figure 4: Bar graph of the wall-to-lumen ratio area of the aortas from control, L-NAME and SHR animals.

be explained by an increase of collagen content or hypertrophy cardiomyocyte. However, newborns L-NAME at 2 days had lower relative cardiac weight probably due to lower number of cardiomyocytes because apoptotic activity increased in the hearts of these animals.

The effect of NO inhibition of the rate of collagen synthesis has been poorly characterized. A study demonstrated that the NO-generation inhibits the protein synthesis in cultured vascular smooth muscle cells [24]. Thus, we observed that NO inhibition is reflected in the rate of synthesis of collagen during neonatal development of the rat heart, especially in L-NAME neonate at 15 days. Therefore, the nitric oxide inhibition in vivo may lead to increase collagen synthesis in the myocardium of the L-NAME newborns.

Reduction in number of elastic lamellae in the aorta of newborns L-NAME and SHR may impair the contractile properties of the aorta. As a possible mechanism we speculate that, in fetuses with growth retardation, such as L-NAME and SHR models, there is impairment in the synthesis of elastin during a critical period of blood vessel development. As a result of the elastin deficiency, the compliance of the conduit arteries is reduced, leading to higher pulse pressure.

Elastin synthesis in the aorta of SHRs was found to exceed control levels in the prehypertensive period, decrease during the development of hypertension and increase again in the period of the established hypertensive state [25] In this study, the number of elastic lamellae increases in L-NAME e SHR neonates at 2 days and latter it stop increasing at 15 days.

The rat offspring born from L-NAME parents, with sustained NO induced hypertension, had a remarkably higher blood pressure [26]. In this study we assess not blood pressure of newborns L-NAME and SHR, and we can not infer at the age of 15 days were hypertensive, however, the synthesis of elastic fibers

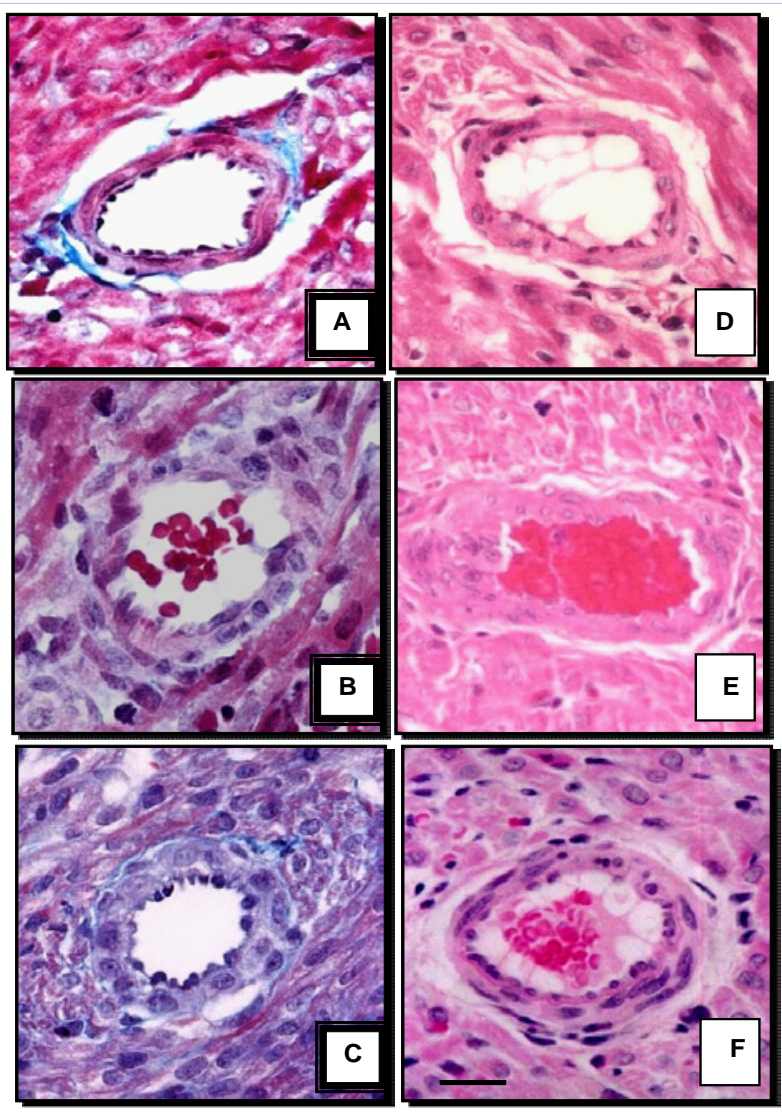

Notes: Control (A and D), L-NAME (B and E) and SHR newborns (C and F) at 2 and 15 post-natal days stained by Mallory's trichrome and hematoxylin-eosin, respectively. A, B and C at 2 days and D, E and F at 15 days. L-NAME and SHR groups had increased wall-to-lumen ratio compared with the control group. Scale bar $=10 \mu \mathrm{m}$

Figure 5: Photomicrographs of the coronary arterioles of control, SHR and L-NAME newborns.

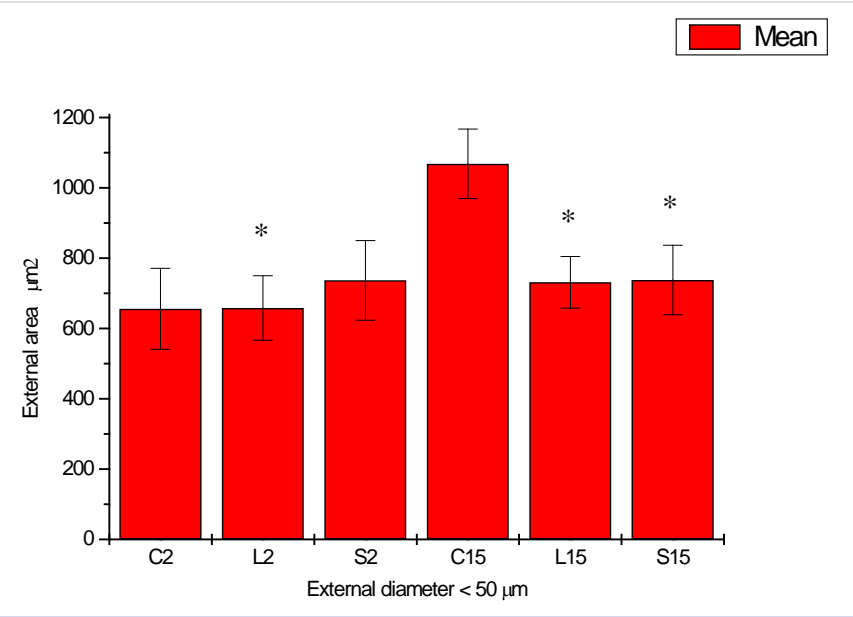

Notes: ${ }^{*} \mathrm{P}<0.05$ vs $\mathrm{C} 15$ by two way ANOVA followed by multiple comparison tests. Abbreviations as Figure 3. Values are means \pm SD; $n$ $=5$ animals per group

Figure 6: Bar graph of the external area of coronary arteries with external diameters $<50 \mu \mathrm{m}$. 


\begin{tabular}{|c|c|c|c|}
\hline Age & Group & Elastic lamellae (n) & Collagen content (\%) \\
\hline \multirow{2}{*}{ 2-day } & Control & $6.05 \pm 0.18$ & $0.611 \pm 0.022$ \\
\cline { 2 - 4 } & L-NAME & $7.10 \pm 0.16^{*}$ & $0.602 \pm 0.020$ \\
\cline { 2 - 4 } & SHR & $7.45 \pm 0.17^{*}$ & $0.743 \pm 0.020^{*}$ \\
\hline \multirow{2}{*}{ 15-day } & & & \\
\cline { 2 - 4 } & Control & $7.60 \pm 0.17$ & $2.118 \pm 0.048$ \\
\cline { 2 - 4 } & L-NAME & $7.00 \pm 0.16$ & $2.251 \pm 0.040$ \\
\cline { 2 - 4 } & SHR & $7.30 \pm 0.19$ & $2.531 \pm 0.040^{*}$ \\
\hline
\end{tabular}

${ }^{b}$ Data are given as means $\pm S D ; n=5$ animals per group ${ }^{*} \mathrm{P}<0.05$ vs controls by two-way ANOVA followed by the multiple comparison test

Table 2: Number of elastic lamellae of the aorta and collagen content of the heart of neonates ${ }^{\mathrm{b}}$.

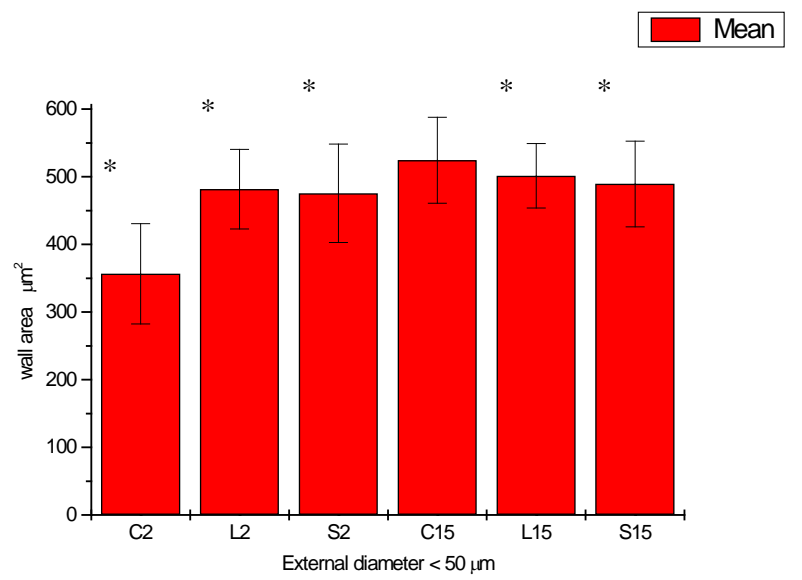

Notes: ${ }^{*} \mathrm{P}<0.05$ vs the corresponding values of the control group at 15 days by two way ANOVA followed by multiple comparison tests. Values are means $\pm S D ; n=5$ animals per group

Figure 7: Bar graph of the wall area of intramyocardial arteries with external diameters $<50 \mu \mathrm{m}$.

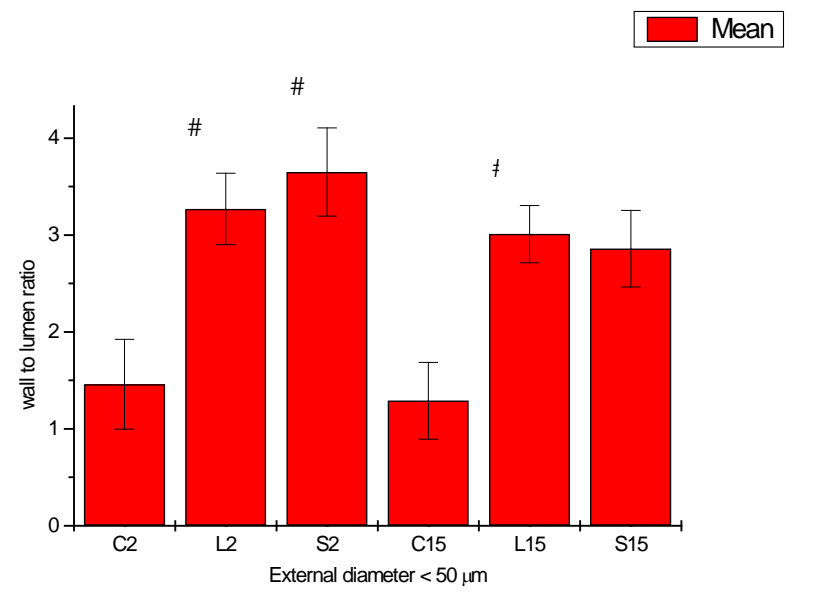

Notes: ${ }^{*} \mathrm{P}<0.05$ vs $\mathrm{C} 2$. ${ }^{*} \mathrm{P}<0.05$ vs $\mathrm{C} 15$ by two way ANOVA followed by multiple comparison tests. Values are means \pm SD; $n=5$ animals per group

Figure 8: Bar graph of the wall-to-lumen ratio of the myocardial microvessels with external diameters $<50 \mu \mathrm{m}$. in the aorta in these animals was similar to that of adult animals before and after the establishment of hypertension.

The modest increase of ratio wall-to-lumen of the aorta in offspring at 2 days from L-NAME-treated mothers in this study could be explained by a high level of NO synthesis and/ or its activity in the wall of major arteries even after L-NAME treatment. This is supported by findings of high endothelial NO production in the pulmonary artery during the fetal and early post-natal period $[27,28]$. NO has been shown to inhibit vascular smooth muscle cell proliferation [24].

The present study showed that the most significant change on the L-NAME neonate myocardium involves the microvasculature, meanly arterioles. The wall-to-lumen ratio of arterioles was significantly higher in the L-NAME and SHR than in the control group at 2 and 15 post-natal days. Numaguchi, K., et al. [8] also found an increase of wall-to-lumen ratio in arterioles and small coronary arterioles on the myocardium of L-NAME-treated rats for 8 weeks. They observed an accumulation of collagen in the media of microvessels and perivascular fibrosis and believed that chronic L-NAME administration might increase sympathetic nerve activity, which may contribute to vascular remodeling. L-NAME-treated rats $(50 \mathrm{mg} / \mathrm{Kg} /$ day $)$ during 8 weeks showed an increase of wall thickness (tunica intima and tunica media) of carotid and coronary arteries in relation to control rats [29]. Using the same dosage during the same period, other authors [30] found a significant increase in the capillary domain in treating animals, suggesting that there is no capillary proliferation, but myocyte hypertrophy during this period.

In spontaneous hypertension, not all vascular beds display the same increase in the ratio between the wall-to-lumen ratio. In SHR, for instance, the change is less marked in the kidney than in the mesenteric, cerebral or hindquarter skeletal muscle vascular bed. Furthermore, not all vessels are affected simultaneously during development [31]. Korsgaard, N., \& Mulvany, M.J. [32] reported a smaller lumen diameter for mesenteric small arteries of hypertensive rats. Also, in subcutaneous resistance arteries of untreated hypertensive patients, a significant reduction of lumen diameter was observed [32].

In established hypertension, peripheral vascular resistance is elevated, primarily due to structural changes in the arterial tree, most notably to an elevation of the wall thickness to lumen diameter ratio of small arteries. According to Poiseuille's law, resistance is more markedly affected by arterial diameter than by the length and number of arteries segments. So, arterial resistance resides primarily in small "resistance-sized" arteries (diameter $<300 \mu \mathrm{m}$ ) and in arterioles with characteristics of precapillary vessels that present only one layer of smooth muscle cells [33]. During the establishment of the arterial hypertension, the cardiac output is normal, but the resistance is elevated $[31,34]$. The arterial structural changes observed in hypertension include an increase in the wall-to-lumen ratio and reduction of the number of microvessels (rarefaction), leading to a reduction 
of the average overall lumen diameter of the arterial system [35]. However, neither arterial hypertrophy nor rarefaction necessarily led to a reduction of lumen diameter and increase in the vascular resistance. Vascular resistance depends on the relationship between wall mass and lumen diameter [36].

Some questions can be rise regarding the increase of wall-to-lumen ratio in the myocardial arterioles of L-NAME neonates in this study: What does promote the increase of the vascular wall thickness: smooth muscle cell hypertrophy and/ or hyperplasia? What factors stimulate the vascular smooth muscle cell hypertrophy and/or hyperplasia? Only the nitric oxide inhibition can promote the arterioles changes or other factors that also contribute to these changes? In general, the smooth muscle hyperplasia cells in the media of hypertensive animals are considered a primary change, whereas hypertrophy is considered a secondary one [37]. In the SHR and Goldblatt hypertensive rats, the media mass increase in the aorta was due to cellular hypertrophy and polyploidy, whereas in rats made hypertensive by aortic coarctation, the median change was due to hyperplasia of the smooth muscle cells [37].

Probably, the myocardial vascular changes induced by long-term blockade of nitric oxide synthesis in rats result from local angiotensin I converting enzyme (ACE) activation. Using immunohistochemistry methods, Takemoto, M., et al. [38] found an increase of ACE in the coronary arteries during the first week of L-NAME administration. These investigators also reported an increase of wall-to-lumen ratio in the myocardial vasculature as well as in the aorta of L-NAME rats.

The increase of the wall-to-lumen ratio in L-NAME neonates at 2 and 15 post-natal days are probably due to the nitric oxide inhibition during the pregnancy joined to arterial pressure increased in the newborns. Both effects induce smooth muscle cell proliferation on myocardial arterioles. It is know that NO inhibits smooth muscle cell proliferation [39]. A decreased NO generation induces the synthesis of growth-promoting factors from the endothelium [40]. The angiotensin-converting enzyme (ACE) activation would increase the formation of angiotensin II, which in turn directly induces vascular smooth muscle proliferation [41] and the release of platelet-derived growth factor [38], transforming growth factor ${ }^{40}$ and endothelins [42]. The findings support the angiotensin II involvement in the vascular smooth muscle cell hypertrophy/hyperplasia during hypertension promoted by the administration of L-NAME in rats.

The majority of nitric oxide synthase (NOS) enzyme in the rat heart is related to vessels and its production by the eighteenth day of the embryo development [43]. Our results point out that nitric oxide inhibition during pregnancy induces vascular smooth muscle cell proliferation and accumulation of collagen in the neonatal hearts.

Not only the vascular smooth muscle development is affected by L-NAME, but also the administration of these nitric oxide inhibitors during pregnancy compromises pyloric musculature, hypertrophy and hyperplasia, in neonate rats [44].
Another factor that may contribute to myocardial microvasculature changes in neonates from L-NAME-treated mothers is the increase in the sympathetic activity in these newborns. In L-NAME-treated rats, the increase in the sympathetic activity leads to vascular remodeling: smooth muscle cell proliferation and lumen occlusion in the myocardial arterioles [8].

\section{Conclusion}

In conclusion, our results demonstrated that the chronic inhibition of NO synthesis with L-NAME during the pregnancy caused microvasculature remodeling in the ages of 2 and 15 days, and increased collagen content in the neonate hearts at $15^{\text {th }}$ post-natal days. These changes were also described in SHR newborns leading similarity between models of hypertension during pregnancy.

\section{References}

1. Noll, G., \& Luscher, T. F. (1998). The endothelium in acute coronary syndromes. European Heart Journal, 19(8 Pt 2), C30-38.

2. King, R. G., Gude, N. M., Di Julio, J. L., \& Brennecke, S. P. (1995). Regulation of human placental fetal vessel tone: role of nitric oxide. Reproduction Fertility and Development, 7(6), 1407-1411.

3. Sladek, S. M., Magness, R. R., \& Conrad K. P (1997). Nitric oxide and pregnancy. American Journal of Physiology, 272(2 Pt 2), R441-R463.

4. Salas, S. P., Altermatt, F., Campos, M., Giacaman, A., \& Rosso, P(1995). Effects of long-term nitric oxide synthesis inhibition on plasma volume expansion and fetal growth in the pregnant rat. Hypertension,26(6 Pt 2), 1019-1023.

5. Zhang, Y., \& Kaufman, S. (2000). Effect of nitric oxide synthase inhibition on cardiovascular and hormonal regulation during pregnancy in the rat. Can J Physiol Pharmacol, 78(5), 423-427.

6. Ribeiro, M. O., Antunes, E., de Nucci, G., Lovisolo, S. M., \& Zatz, R. (1992) Chronic inhibition of nitric oxide synthesis. A new model animal of arterial hypertension. Hypertension, 20(3), 298-303.

7. Arnal, J. F., el Amrani, A.I., Chatellier, G., Menard, J.,\& Michel J.B(1993). Cardiac weight in hypertension induced by nitric oxide synthase blockade. Hypertension, 22(3), 380-387.

8. Numaguchi, K., Egashira, K., Takemoto, M., Kadokami, T., Shimokawa, H., Sueishi, K., Takeshita A.(1995). Chronic inhibition of nitric oxide synthesis causes coronary microvascular remodeling in rats. Hypertension, 26(6 Pt 1),957-962.

9. Pessanha, M.G., Mandarin-de-Lacerda, C.A., Hahn, M.D.(1999). Stereologyandimmunohistochemistryof the myocardium in experimental hypertension: long-term and low-dosage administration of inhibitor of the nitric oxide synthesis. Pathobiology, 67(1), 26-33.

10. Mandarim-de-Lacerda, C.A., \& Pereira, L.M.M.( 2000). Numerical density of cardiomyocytes in chronic nitric oxide synthesis inhibition. Pathobiology,68(1), 36-42.

11. de Oliveira, C.F., Cintra, K.A., Teixeira, S.A., De Luca I.M.S, Antunes E., \& De Nucci G. (2000). Development of cardiomyocyte hypotrophy in rats under prolonged treatment with a low dose of a nitric oxide synthesis inhibitor. European Journal of Pharmacology,391(1-2), 121-126.

12.Miller, M.J.S, Voelker, C. A., Olister, S., Thompson, J. H., Zhang, X.-J., 
Rivera, D. (1996). Fetal growth retardation in rats may result from apoptosis: role of peroxynitrite. Free Radical Biology and Medicine 21(5), 619-629.

13. Diket, A. L., Pierce, M. R., Upender, K. M., Voelker, S.S., Eloby-Childress, S., Greenberg, S. S., et al. (1994). Nitric oxide inhibition causes intrauterine growth retardation and hind-limb disruptions in rats. American Journal of Obstetrics and Gynecology 171(5), 1243-1250.

14. Pierce, L. R., Pierce, M. R., Liu, H., Kadowitz, P. J., \& Miller, M. J. S (1996). Limb reduction defects after prenatal inhibition of nitric oxide synthase in rats. Pediatric Research 38(6), 905-911.

15. Lee, T. C., Zhao, Y. D., Courtman, D. W., \& Stewart, D. J. (2000). Abnormal aortic valve development in mice lacking endothelial nitric oxide synthase. Circulation 101(20), 2345-2348.

16. Feng, Q., Song, W., Lu X., Hamilton, J.A., Lei, M. L., Peng, T.,\& Yee, S-P. (2000). Development of heart failure and congenital septal defects in mice lacking endothelial nitric oxide. Circulation 106(7), 873-879.

17. Hefler, L. A., Reyes, C. A., O’Brien, W. E., \& Gregg , A. R. (2001). Perinatal development of endothelial nitric oxide synthase-deficient mice. BIOLOGY OF REPRODUCTION 64(2), 666-673.

18. Bloch, W., Fleischmann, B. K., Lorke, D. E., Andressen, C., Hops, B. Hescheler, J., et.al.(1999). Nitric oxide synthase expression and role during cardiomyogenesis. Cardiovasc Res 43(3), 675-684.

19. Whittaker, P., Kloner, R. A., Boughner, D. R., Pickering, J. G.(1994). Quantitative assessment of myocardial collagen with picrosirius red staining and circularly polarized light. Basic Res Cardiol, 89(5), 397410.

20. Gardiner, S. M., Compton, A. M., Bennett, T., Palmer, R. M. J., Moncada, S.(1990). Regional haemodynamic changes during oral ingestion of NG-monomethyl-L-arginine or NG-nitro-L-arginine methyl ester in conscious Brattleboro rats. Br J Pharmacol ,101(1), 10-12.

21.Gregg, A. R., Schauer, A., Shi O., Liu Z., Lee C. G. L., O’Brien W.E.(1998) Limb reduction defects in endothelial nitric oxide synthase-deficient mice. Am J Physiol Heart Circ Physiol 1998, 275(6 Pt 2), H2319H2324.

22. Moreau, P., Tea, B.-S., Dam, T. V., Hamet, P.(1997). Altered balance between cell replication and apoptosis in hearts and kidneys of newborn SHR. Hypertension ,30(3 Pt 2), 720

23. Kajstura, J., Mansukhani, M., Cheng, W. (1995). Programmed cell death and expression of the protooncogene bcl-2 in myocytes during postnatal maturation of the heart. Exp Cell Res ,219(1), 110-121.

24. Kolpakov, V., Gordon, D., Kulik, T.J. (1995). Nitric oxide-generating compounds inhibit total protein and collagen synthesis in cultured vascular smooth muscle cells. Circ Res ,76(2), 305-309.

25. Deyl, Z., Jelinek, J., Macek, K., Chadakov, G., \& Vankov, V.N.(1987) Collagen and elastin synthesis in the aorta of spontaneously hypertensive rats. Blood Vessels 24, 313-320.

26. Gerová, M., Bernátová, I., Török, J., \& Jurani, M. (2002). Cardiovascular system in offsprings of hypertensive rats with defective nitric oxide production. Physiological Research,51(5), 465-474.

27. Abman, S. H., Cathfield, B. A., Rodman, D. M., Hall, S. L., \& McMurtry, I. F.,(1991). Maturational changes in endothelium-derived relaxing factor activity of ovine pulmonary arteries in vitro. American Journal of Physiology,260(4 Pt 1), L280-285.
28.Shaul, P. W., Farrar, M. A., \& Magness, R. R.,(1993). Pulmonary endothelial nitric oxide production is developmentally regulated in the fetus and newborn. American Journal of Physiology, 265(4 Pt 2), 1056-1063.

29. Kristek, F., \& Gerová, M. (1996). Long-term NO synthase inhibition affects heart weight and geometry of coronary and carotid arteries. Physiol Res ,45(5),361-367.

30.Sládek, T., Gerová, M., Znojil, V., \& Devát L.(1996). Morphometric characteristics of cardiac hypertrophy induced by long-term inhibition of NO synthase. Physiological Research , 45(4), 335-338.

31. Mulvany, M. J., \& Aalkajaer, C.(1990). Structure and function of small arteries. Physiological Reviews, 70(4), 921-961.

32. Korsgaard, N., \& Mulvany, M. J.(1998). Cellular hypertrophy in mesenteric resistance vessels from renal hypertensive rats. Hypertension, 12(2), 162-167.

33. Korner, P.I. (1995). Cardiovascular hypertrophy and hypertension: causes and consequences. Blood Press Suppl, 2,6-16.

34. Korner, P.J., Angus, J. A.(1992). Structural determinants of vascular resistance properties in hypertension. Hemodynamic and model analysis. J Vasc Res, 29(4), 293-312.

35. Boudier, H. A. (1999). Arteriolar and capillary remodelling in hypertension. Drugs, 58, 37-40.

36. Heagerty, A. M., Aalkjaer, C., Bund, S. J., Korsgaard, N., \& Mulvany, M.J.(1993). Small artery structure in hypertension. Dual processes of remodeling and growth. Hypertension, 21(4), 391-397.

37. Smeda, J. S., Lee, R. M., \& Forrest, J. B.(1998).Structural and reactivity alterations of the renal vasculature of spontaneously hypertensive rats prior to and during established hypertension. Circulation Research, 63, 518-533.

38. Takemoto, M., Egashira, K., Usui, M., Numaguchi, K., Tomita, H., Tsutsui H., et al.(1997).Important role of tissue angiotensin-converting enzyme activity in the pathogenesis of coronary vascular and myocardial structural changes induced by long-term blockade of nitric oxide synthesis in rats. J Clin Invest 99(2), 278-287.

39. Busse, R., \& Fleming, I.,(1995). Regulation and functional consequences of endothelial nitric oxide production. Annals of Internal Medicine, $27(3), 331-340$.

40. Kourembanas, S., McQuillan, L. P., Leung, G. K., \& Faller, D. V,(1993). Nitric oxide regulates the expression of vasoconstrictors and growth factors by vascular endothelium under normoxia and hypoxia. The Journal of Clinical Investigation, 92(1), 99-104.

41. Itoh, H., Mukoyama, M., Pratt, R. E., Gibbons, G. H., \& Dzau, V. J.,(1999). Multiple autocrine growth factors modulate vascular smooth muscle cell growth response to angiotensin II. J Clin Invest 91(5), 2268-2274.

42. Lüscher, T. F., \& Noll, G. Is it all in the genes...? Nitric oxide synthase and coronary vasospasm. Circulation 99(22), 2855-2857.

43. Ursell, P. C., \& Mayes, M. (1996). Endothelial isoform of nitric oxide synthase in rat heart increases during development. Anatomical record, 246(4), 465-472.

44.Voelker, C. A., Miller, M. J. S., Zhang X.-J., Childress E., Clark D. A., \& Pierce M.R.(1995). Perinatal nitric oxide synthase inhibition retards neonatal growth by inducing hypertrophic pyloric stenosis in rats. Pediatric Research, 38(5), 768-774. 